\title{
Drinking Water Treatment Plant Design Incorporating Variability and Uncertainty
}

\author{
Dominic L. Boccelli ${ }^{1}$; Mitchell J. Small ${ }^{2}$; and Urmila M. Diwekar ${ }^{3}$
}

\begin{abstract}
Both inherent natural variability and model parameter uncertainty must be considered in the development of robust and reliable designs for drinking water treatment. This study presents an optimization framework for investigating the effects of five variable influent parameters and three uncertain model parameters on the least-cost treatment plant configuration (contact, direct, or nonsweep conventional filtration) that reliably satisfies an effluent particulate matter concentration constraint. Incorporating variability and uncertainty within the decision-making framework generates information for investigating: (1) impacts on total cost and treatment reliability; (2) shifts on the least-cost treatment configuration for providing reliable treatment; and (3) the importance of the individual variable and uncertain parameter distributions for reliably satisfying an effluent water quality constraint. Increasing the magnitude of influent variability and model parameter uncertainty results in a greater expected design cost due, generally, to increases in process sizing required to reliably satisfy the effluent concentration constraint. The inclusion of variability and uncertainty can also produce a shift in the locations of the least-cost configuration regions, which are dependent on the expected influent water quality and the magnitude of variability and uncertainty. The additional information provided by incorporating the variable and uncertain parameters illustrates that parameter distributions related to the primary removal mechanism are critical, and that contact and direct filtration are more sensitive to variability and uncertainty than conventional filtration.
\end{abstract}

DOI: 10.1061/(ASCE)0733-9372(2007)133:3(303)

CE Database subject headings: Potable water; Water treatment plants; Stochastic processes; Optimization; Uncertainty principles.

\section{Introduction and Objectives}

Decision making related to drinking water treatment is, in general, a complex process that must satisfy multiple treatment objectives through the design of integrated treatment and waste processes while constraining costs. Additionally, there is inherent variability that must be considered, yet cannot be controlled (at least not easily), such as flow rate, influent water quality concentrations, and temperature. The effects of these variations must be considered to ensure that the effluent water quality reliably satisfies the appropriate regulatory statutes. More formalized approaches that utilize mathematical models to assist in the design process add additional complexities. The majority of process models require some of the model parameters to be estimated using experimental or field data, or gathered from expert elicita-

\footnotetext{
${ }^{1}$ Office of Research and Development, National Homeland Security Research Center, U.S. Environmental Protection Agency, MS 163, 26 W. Martin Luther King Dr., Cincinnati, OH 45268 (corresponding author). E-mail: boccelli.dominic@epa.gov

${ }^{2}$ H. John Heinz III Professor of Environmental Engineering, Dept. of Civil and Environmental Engineering and Dept. of Engineering and Public Policy, Carnegie Mellon Univ., Pittsburgh, PA 15213.

${ }^{3}$ President, Center for Uncertain Systems: Tools for Optimization and Management, Vishwamitra Research Institute, 34 North Cass Ave., Westmont, IL 60559.

Note. Discussion open until August 1, 2007. Separate discussions must be submitted for individual papers. To extend the closing date by one month, a written request must be filed with the ASCE Managing Editor. The manuscript for this paper was submitted for review and possible publication on October 19, 2005; approved on June 14, 2006. This paper is part of the Journal of Environmental Engineering, Vol. 133, No. 3, March 1, 2007. CASCE, ISSN 0733-9372/2007/3-303-312/ $\$ 25.00$.
}

tion, which introduce model uncertainty into the design process. Finally, uncertainty in the structure of the model representing the integrated treatment process can introduce additional complexity, although this is more difficult to incorporate into the decisionmaking process.

The use of formalized decision-making tools for the investigation of integrated drinking water treatment plant design has been relatively rare, with most studies focusing on the removal of particulate matter from the raw water stream. Wiesner et al. (1987) investigated the regions of least-cost configurations of rapid mix, flocculation, sedimentation, and filtration for seven different influent conditions for particulate removal, which were shown to be relatively consistent with existing treatment plant configurations (Wiesner and Mazounie 1989). Wu and Chu (1991) utilized simplified empirical treatment process models and data from an existing treatment plant and developed treatment plant designs similar to the existing plant. Mhaisalkar et al. (1993) solved the design problem using dynamic programming and simplified process models for flocculation and sedimentation with monodisperse particle suspensions. Dharmappa et al. (1994) performed a study similar to that of Wiesner et al. (1987) that included waste handling options for three different particle size distributions. Boccelli et al. (2004) extended the study of Wiesner et al. (1987) to include a wider range of influent conditions, and examined the changes in least-cost configuration regions under different flow rate and particle density conditions.

All of these studies assumed that the influent water quality conditions, flow rate, and model parameters were fixed and known - that is, there was no variability or uncertainty included in the decision-making process. While studies that explore a wide range of influent conditions and model parameter values (e.g., Wiesner et al. 1987; Boccelli et al. 2004) illustrate the impact 
of changing an individual parameter value on process design and configuration, these deterministic studies are inadequate for assessing the overall impact of variability and uncertainty on these designs and configurations. Mhaisalkar et al. (1993) provided some insight into the potential effects of variability and uncertainty by performing a series of sensitivity analyses on the optimal deterministic solutions that showed the designs to be relatively insensitive to changes in flow rate, influent particle concentration, and cost equation parameters. Their study also discussed the importance of including flow and influent water quality variability and uncertainty in the decision-making process for providing reliable treatment.

This study presents a framework for incorporating influent variability and model parameter uncertainty in the design process, which is illustrated using an integrated treatment model for particulate removal based on a rectilinear approach (Lawler et al. 1980; Ramaley et al. 1981; Wiesner et al. 1987) as an example application. Model structure uncertainty, which certainly exists in this application, is not explored in this study. However, the framework is sufficiently general to allow model structure uncertainty or the inclusion of additional process models to be evaluated.

The impact of variability and uncertainty is evaluated with respect to the expected design cost, distribution of effluent particulate concentrations, and value of the stochastic solution. More importantly, the inclusion of variable and uncertain parameters is used to explore the potential shift in the least-cost regions of three different treatment configurations (contact, direct, and nonsweep conventional filtration). The solution framework also generates information that allows the determination of the important variable and uncertain parameter distributions with respect to reliably satisfying the effluent particulate concentration constraint. The explicit inclusion of variability and uncertainty in the design framework is important for understanding the potential effects on treatment plant design and selection and for identifying important parameters that may require additional characterization or improved estimation.

\section{Process Models}

The change in the particle size distribution and concentration through rapid mix, flocculation, sedimentation, and filtration are described using the rectilinear approach (Lawler et al. 1980; Ramaley et al. 1981; Wiesner et al. 1987) under nonsweep, or charge neutralization, treatment conditions. While other approaches have been formulated for representing flocculation and sedimentation [e.g., curvilinear (Han and Lawler 1991, 1992) and fractal (Lee et al. 2000; Li and Logan 1997, 2000; Wiesner 1992) approaches], the rectilinear approach has been shown, as an integrated model, to reasonably represent actual treatment plant configurations (Wiesner and Mazounie 1989). However, these models are not entirely mechanistic and make use of empirical coefficients to account for other factors, such as surface charge and $\mathrm{pH}$, not explicitly included in the process models. The following briefly describes the process models and statistical distributions used to formulate the problem; complete details may be found in Boccelli (2003).

The influent particle size distribution is described by a powerlaw distribution (Lawler et al. 1980)

$$
\frac{d N}{d \log \left(d_{p}\right)}=2.3 A d_{p}^{(1-\beta)}
$$

where $N=$ number concentration of particles; $d_{p}=$ particle diameter; $A=$ parameter related to the particle concentration; and $\beta=$ parameter related to the shape of the particle size distribution. The change in particle size distribution through rapid mix, flocculation, and sedimentation is described using Smoluchowski's equations (Amirtharajah and O'Melia 1990; Lawler et al. 1980). Removal of particles during sedimentation is incorporated using a term for Stokes' settling (Lawler et al. 1980; Wiesner et al. 1987) with a correction factor for drag on large particle sizes (J. M. Montgomery 1985). The rapid mix, flocculation, and sedimentation processes utilize a particle collision efficiency, $\alpha$, that empirically accounts for the impact of water chemistry and coagulant type on particulate agglomeration. For this study, the expected value of $\alpha$ is set to 0.4 (Wiesner et al. 1987). Numerically, the continuous influent particle size distribution [Eq. (1)] is represented by a logarithmic discretization of particle sizes into 51 discrete particle diameters using $\Delta \log \left(d_{p}\right)=0.04$ [as in Ramaley et al. (1981), additional bins are included, and initially left empty, to account for the formation of particles with diameters up to $300 \mu \mathrm{m}]$. To represent settling, the sedimentation tank is discretized into seven layers, which are coupled through the settling term. The resulting set of ordinary differential equations (63-88 for rapid mix and flocculation, and 441-616 for sedimentation; dependent on the influent size range) are a stiff set of ordinary differential equations (ODEs) solved using LSODE (Hindmarsh 1983).

The filtration model used in this study was developed by O'Melia and Ali (1978) and utilizes the volume averaged particle diameter, $\left(\bar{d}_{p}\right)_{V}$, and particulate concentration leaving the sedimentation tank, $C_{f}$, as inputs to the filtration model. The filter media properties for this study consist of a media diameter, $d_{m}$, of $0.1 \mathrm{~cm}$ and a clean-bed porosity, $\epsilon$, of 0.36 . The collection efficiency of the sand media, $\alpha_{f}$, and entrapped particles, $\alpha_{f, p}$, are 0.76 and 0.08 , respectively (Wiesner et al. 1987). As with $\alpha$, the $\alpha_{f}$ and $\alpha_{f, p}$ parameters empirically account for the impact of water chemistry and filter media properties on particulate removal.

The cost equations used to represent the capital and operating and maintenance (OM) costs are taken from Clark (1982) and a modified form of the equations presented by Letterman (1980) to describe filtration OM costs (i.e., Wiesner et al. 1987). Costs are updated to 1997 dollars and amortized over 20 years at an interest rate of $8 \%$. The total cost is presented as the annualized capital cost plus the annual OM costs.

This study explicitly considers five variable parameters (flow rate, $Q$; temperature, $T$; influent particle concentration, $C$; distribution shape parameter, $\beta$; and density, $\rho$ ) and three uncertain model parameters $\left(\alpha, \alpha_{f}\right.$, and $\left.\alpha_{f, p}\right)$. Table 1 presents the average values and distributions used to represent the variable and uncertain parameters. The average values are based on data from Lawler et al. (1980), Wiesner (1985), and Wiesner et al. (1987). Three different standard deviation values are used in this study based on coefficient of variation $(\mathrm{COV}=\sigma / \mu)$ values of 0.016 , 0.032 , and 0.081 , where $\sigma=$ standard deviation and $\mu=$ average value presented in Table 1 . The COV values of $0.016,0.032$, and 0.081 were selected such that $99.9 \%$ of the simulated values are within $\pm 5,10$, and $25 \%$ of $\mu$, respectively, for the normally distributed parameters. Given the potential range of influent water quality characteristics from different source waters and the limited data associated with model parameter values collected in practice, the individual COV values are applied simultaneously to all of the variable and uncertain parameters and are intended to illustrate the potential impacts of variability and uncertainty magnitude on the design decisions, not to represent the conditions at 
Table 1. Variable and Uncertain Parameters, Average Values, and Distributions for Determining Optimal Treatment Plant Designs Using Optimization under Uncertainty

\begin{tabular}{lccccc}
\hline Uncertain parameter & Average & Distribution & Variable parameter & Average & Distribution \\
\hline Collision efficiency $\alpha$ & 0.40 & Beta $(\alpha, \beta, 0,1)$ & Flow rate $Q(\mathrm{MGD})$ & 10 & Normal $(\mu, \sigma)$ \\
Collection efficiency: Media $\alpha_{f}$ & 0.76 & Beta $(\alpha, \beta, 0,1)$ & Concentration $C(\mathrm{mg} / \mathrm{L})^{\mathrm{a}}$ & $4,12,32,80,128$ & Normal $(\mu, \sigma)$ \\
Collection efficiency: Particle $\alpha_{f, p}$ & 0.08 & Beta $(\alpha, \beta, 0,1)$ & Temperature $T\left({ }^{\circ} \mathrm{C}\right)$ & 20 & Normal $(\mu, \sigma)$ \\
& & & Shape parameter $\beta$ & $4,3,5^{\text {b }} 5^{\mathrm{b}}$ & Normal $(\mu, \sigma)$ \\
& & & Density $\rho\left(\mathrm{g} / \mathrm{cm}^{3}\right)$ & 1.20 & Beta $(\alpha, \beta, 1.05,2.40)$ \\
\hline
\end{tabular}

Note: For size ranges $0.10-10.0,0.25-25.0,0.50-50.0,0.75-75.0$, and $1.0-100.0 \mu \mathrm{m}$. Beta distributions include minimum and maximum limits on the distribution. [Parameters are based on Lawler et al. (1980), Wiesner (1985), and Wiesner et al. (1987).]

${ }^{\mathrm{a}}$ Additional values of $\mathrm{C}$ evaluated where necessary.

${ }^{\mathrm{b}}$ Only for influent size ranges of $0.25-25.0$ and $0.75-75.0 \mu \mathrm{m}$.

any particular plant. The parameters of the normal distributions are simply the average and standard deviation values presented. The parameters of the beta distributions, $\alpha$ and $\beta$, are determined using method-of-moment equations with the appropriate mean and standard deviation values (DeGroot and Schervish 2002). The additional arguments given for the beta distributions represent the minimum and maximum allowable values of the variable or uncertain parameter.

\section{Why Include Variability and Uncertainty?}

The inclusion of variability and uncertainty in the modeling framework is an approach for representing the potential range of behavior that might be observed within an actual process. The traditional inclusion of "safety factors" in the design process is intended to account for these variabilities and uncertainties by overdesigning the individual processes. By explicitly including variability and uncertainty in the design process, additional information is provided to the problem, allowing a design to be selected that provides adequate removal over the range of conditions at a minimum cost. Fig. 1 illustrates the effect of simulating variability and uncertainty on the effluent concentration using a deterministic least-cost design (no "safety factor") for nonsweep conventional treatment with an influent concentration of $80 \mathrm{mg} / \mathrm{L}, \beta=4$, and particle size range of $0.25-25.0 \mu \mathrm{m}$ (Boccelli et al. 2004). The solid line represents the regulatory limit $(0.6 \mathrm{mg} / \mathrm{L})$ that is satisfied in the deterministic case; the

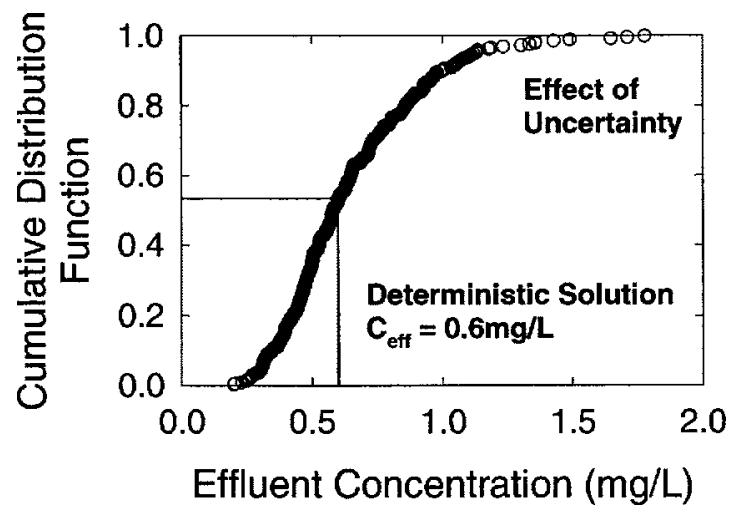

Fig. 1. Distribution of effluent particulate concentrations resulting from simulating variability and uncertainty with an optimal deterministic solution. Solid line represents the deterministic effluent particulate value of $0.6 \mathrm{mg} / \mathrm{L}$ (average influent concentration of $80 \mathrm{mg} / \mathrm{L}$ and influent size range of $0.25-25.0 \mu \mathrm{m})$. distribution of effluent concentrations-the cumulative distribution function-is a result of simulating variability and uncertainty with the deterministic solution and represents the probability (Pr) that the effluent concentration is less than $x, \operatorname{Pr}(X \leq x)$ $(\mathrm{COV}=0.081$ for all variable and uncertain parameters $)$. For this example, approximately $47 \%$ of the simulated samples violate the effluent concentration constraint of $0.6 \mathrm{mg} / \mathrm{L}[1-\operatorname{Pr}(X \leq x)]$, and result in effluent particle concentrations up to three times greater than the effluent concentration constraint. Thus, the additional information provided by including variability and uncertainty can have a profound impact on the performance of a deterministic design, which can be included in the solution methodology allowing designs to be developed that are more reliable with respect to satisfying the various constraints.

\section{Solution Methodology}

The explicit inclusion of variability and uncertainty in the design process results in the following general optimization problem formulation:

$$
\min _{x} E[f(x, \xi)]
$$

subject to

$$
\begin{aligned}
& h(x, \xi)=0 \\
& g(x, \xi) \leq 0
\end{aligned}
$$

where the expected cost function, $E[f(x, \xi)]$, is to be minimized subject to constraints affected by the variable and uncertain parameters, $\xi$, where $\xi=\left[\xi_{1}, \xi_{2}, \ldots, \xi_{k}\right]$, and $\xi_{i}=n$-dimensional array containing $n$ realizations of the $j$ th variable or uncertain parameter, $j=1, \ldots, k$. The functions $h(x, \xi)$ and $g(x, \xi)$ can take on deterministic and stochastic formulations. Stochastic formulations can be classified as robust, based on a central measure, e.g., $E[y(x, \xi)]$; or reliable, satisfying a constraint at some confidence level, $\operatorname{Pr}[y(x, \xi) \leq Y] \geq \gamma$ (Eldred et al. 2002). Evaluating probabilistic quantities is typically performed using numerical integration. Two common numerical approaches are Monte Carlo-type integration (e.g., Chaudhuri and Diwekar 1996) and quadrature formulations (e.g., Straub and Grossman 1993). The interested reader is referred to Pistikopoulos (1995) and Acevedo and Pistikopoulos (1998) for a review on problem formulation and solution techniques for process design under uncertainty.

The solution of the nonlinear optimization under uncertainty problem is solved in two loops (e.g., Diwekar and Kalagnanam 1997). An outer loop contains a nonlinear programming optimization routine [sequential quadratic programming (SQP)] (Biegler 
and Cuthrell 1985), which passes the decision variables to a stochastic modeler, and generates the necessary gradient information. An inner loop simulates the $k$ variable and uncertain distributions using Halton sequence samples, a low-discrepancy Monte Carlo-type sampling technique, based on the algorithm of Diwekar and Kalagnanam (1997), by generating $n$ samples from the $k$ probability distributions. The algorithm of Diwekar and Kalagnanam (1997) generates a $k+1$-dimensional set of uncertain variables using Hammersley sequence samples, $x=\left[(i-1) / n, \phi_{1}(i), \phi_{2}(i), \ldots, \phi_{k}(i)\right], \quad i=1, \ldots, n$, which gives rise to a $k$-dimensional Halton sequence when the first column of $x$ is removed (see de Hoop and Spencer 1996, Appendix B, for a brief explanation). Each of these individual $n$ samples are used in a forward modeling approach to estimate the individual objective and constraint values. This set of $n$ objective and constraint values is utilized to estimate the various probabilistic measures through numerical integration.

Within the inner loop, statistical measures, such as the mean of $y$

$$
E[y(x, \xi)]=\frac{\sum_{i=1}^{n} y_{i}\left(x, \xi_{i}\right)}{n}
$$

can be readily calculated. For other statistical measures, such as reliability constraints

$$
\operatorname{Pr}[y(x, \xi) \leq Y] \geq \gamma
$$

the calculation is not as straightforward. A very simple method for estimating the probability in Eq. (3) is to let $\operatorname{Pr}[y(x, \xi) \leq Y]=1-n_{\text {viol }} / n$, where $n_{\text {viol }}=$ number of individual samples that violate the constraint $\left[y_{i}\left(x, \xi_{i}\right)>Y\right]$; and $n=$ total number of samples. This formulation, however, is noncontinuous and nonsmooth and can cause computational difficulties associated with determining a search direction through the perturbation analysis of the SQP algorithm.

Instead, the critical value of $y(x, \xi), y_{c}$ is considered, resulting in the following constraint:

$$
y_{c}=F^{-1}(\gamma) \leq Y
$$

where $F=$ cumulative distribution function (cdf) for $y$. Unfortunately, the distribution of $y$ is not generally known a priori. Therefore, $y_{c}$ must be estimated using the $n$ simulated values of $y_{i}\left(x, \xi_{i}\right)$ with an empirical cdf by

1. Sorting the values of $y_{i}\left(x, \xi_{i}\right), i=1, \ldots, n$ in descending order;

2. Determining the critical array index, $I_{c}$, given by $\lfloor n(1-\gamma)\rfloor+1$; and

3. Estimating $y_{c}=y_{\text {sorted }}\left[I_{c}\right]$.

This formulation ensures that $1-n_{\text {viol }} / n \geq \gamma$. This formulation is now continuous, yet nonsmooth, and allows the problem to be solved using the SQP algorithm.

\section{Problem Formulation}

There are three different treatment configurations considered for determining the least-cost treatment design for particulate removal: contact, direct, and nonsweep conventional filtration. Each configuration utilizes a combination of the individual treatment processes of rapid mix, flocculation, sedimentation, and filtration. Table 2 shows the individual treatment processes included in each of the treatment configurations. For each set of influent conditions
Table 2. Individual Treatment Processes of Rapid Mix, Flocculation, Sedimentation, and Filtration Included in Three Different Filtration Treatment Configurations

\begin{tabular}{lcccc}
\hline $\begin{array}{l}\text { Treatment } \\
\text { configuration }\end{array}$ & $\begin{array}{c}\text { Rapid } \\
\text { mix }\end{array}$ & Flocculation & Sedimentation & Filtration \\
\hline Contact & $\mathrm{X}$ & & & $\mathrm{X}$ \\
Direct & $\mathrm{X}$ & $\mathrm{X}$ & & $\mathrm{X}$ \\
Conventional & $\mathrm{X}$ & $\mathrm{X}$ & $\mathrm{X}$ & $\mathrm{X}$ \\
\hline
\end{tabular}

considered, the least-cost design for each of the three treatment configurations is determined. The configuration with the overall lowest cost is then selected as the least-cost option.

Since variability and uncertainty are included in the optimization, the objective is to minimize the expected cost for each of the three configurations. The formulation of the optimization problem is as follows:

$$
\min _{x} E\left[\sum \mathrm{CC}+\sum \mathrm{OM}\right]
$$

Subject to process constraints

$$
\begin{gathered}
\operatorname{Pr}\left(t_{F} \geq 15 \mathrm{~min}\right) \geq \gamma \quad \text { and } \operatorname{Pr}\left(t_{F} \leq 60 \mathrm{~min}\right) \geq \gamma \\
10 \leq G_{F} \leq 50 \mathrm{~s}^{-1}\left(75 \mathrm{~s}^{-1} \text { for direct }\right) \\
\operatorname{Pr}\left(0.5 \mathrm{~h} \leq t_{s}\right) \geq \gamma
\end{gathered}
$$

$$
\operatorname{Pr}\left[L_{f} \geq 6 \mathrm{~L} /\left(\mathrm{m}^{2} \mathrm{~min}\right)\right] \geq \gamma \quad \text { and } \operatorname{Pr}\left[L_{f} \leq 480\left(\mathrm{~L} / \mathrm{m}^{2} \mathrm{~min}\right)\right] \geq \gamma
$$

and integrated constraints

$$
\begin{gathered}
\operatorname{Pr}\left(C_{\text {eff }} \leq 0.6 \mathrm{mg} / \mathrm{L}\right) \geq \gamma \\
h_{f} \leq 250 \mathrm{~cm} \\
0 \leq E[\mathrm{RR}] \leq 0.15 \\
\operatorname{Pr}\left(t_{f} \geq 12 \mathrm{~h}\right) \geq \gamma \quad \text { and } \operatorname{Pr}\left(t_{f} \leq 168 \mathrm{~h}\right) \geq \gamma
\end{gathered}
$$

which minimizes the expected annual design cost [sum of the annualized capital cost, $\mathrm{CC}(x, \xi)$, and operation and maintenance, $\operatorname{OM}(x, \xi)$, cost] by altering the decision variables, $x$, subject to individual process and integrated constraints. The decision variables for the treatment processes are flocculation process volume, $V_{F}\left[\mathrm{~L}^{3}\right]$, and mixing intensity, $G_{F}\left[\mathrm{~T}^{-1}\right]$, and sedimentation and filtration process area, $A_{s}$ and $A_{f}\left[\mathrm{~L}^{2}\right]$. Based on a previous study (Boccelli 2003), four additional design values were fixed: the rapid mix tank is designed with a retention time $\leq 0.1 \mathrm{~s}$ in $95 \%$ of the samples and a mixing intensity of $700 \mathrm{~s}^{-1}$; the sedimentation depth was set at $5.0 \mathrm{~m}$; and the filter depth set at $90 \mathrm{~cm}$. Process constraints are considered on flocculation residence time, $t_{F}=V_{F} / Q[\mathrm{~T}]$, and mixing intensity, $G_{F}\left[\mathrm{~T}^{-1}\right]$; sedimentation residence time, $t_{s}=\left(A_{s} h\right) / Q[\mathrm{~T}]$; and filtration loading rate, $L_{f}=Q / A_{f}\left[\mathrm{~L}^{3} / \mathrm{L}^{2} \mathrm{~T}\right]$ (Montgomery 1985; Kawamura 2000; Wiesner et al. 1987). Integrated constraints are considered on effluent particulate concentration, $C_{\text {eff }}(x, \xi)$, filtration headloss, $h_{f}(x, \xi)$, recycle ratio (the fraction of total flow used in the backwash process), $\operatorname{RR}(x, \xi)$, and filter run time, $t_{f}(x, \xi)$. The integrated constraints are implicitly functions of the individual processes and are the reason the individual treatment processes must be considered simultaneously in the decision-making process. 
The constraint set is a mix of deterministic and stochastic constraints. The individual process constraints [Eqs. (6)-(9)], with the exception of $G_{F}$, are all reliability constraints. The individual retention time and loading rate process constraints are only functions of $Q$ and can be transformed into a deterministic equivalent constraint. For the integrated constraints [Eqs. (10)-(13)], the headloss constraint is a deterministic constraint as the filters are assumed to backwash when the headloss reaches $250 \mathrm{~cm}$; the recycle ratio is a robustness constraint; and the effluent particulate concentration and filter run time constraints are both formulated as reliability constraints. Current regulations for turbidity state that the combined filter effluent must be $\leq 0.3$ nephelometric turbidity units (NTU) in 95\%, or more, of the monthly samples (USEPA 1998). Assuming that $2.0 \mathrm{mg} / \mathrm{L}$ of particulate matter is equivalent to 1.0 NTU (Wiesner et al. 1987), the regulatory constraint on $C_{\text {eff }}$ is $0.6 \mathrm{mg} / \mathrm{L}$ and is not to be exceeded in more than $5 \%$ of the samples (i.e., $\gamma=0.95$ ). $C_{\text {eff }}$ is determined by averaging the effluent concentration values from $n_{\text {filt }}$ filters assuming staggered backwashing, where $n_{\text {filt }}=1.2 E[Q]^{0.5}$, rounded up (Kawamura 2000). The various reliability constraints can be satisfied at varying confidence levels. However, for this study all reliability constraints were satisfied with a $95 \%$ confidence level $(\gamma=0.95)$. The resulting problem has four decision variables and 13 bounds and constraints for the conventional treatment problem. For each of the conditions present in Table 1, solutions are generated for each individual COV value applied to all variable and uncertain parameters and five different influent particle size ranges $(0.10-10.0,0.25-25.0,0.50-50.0,0.75-75.0$, and $1.0-100.0 \mu \mathrm{m})$.

\section{Results}

To develop a reliable treatment plant design with respect to particulate removal, all of the variable and uncertain parameters are simultaneously incorporated into the decision-making framework. The results are analyzed by evaluating: (1) the cost and value of the stochastic solution; (2) the effect of variability and uncertainty on the least-cost treatment configuration regions; and (3) the importance of the variable and uncertain parameter distributions with respect to effluent particulate concentration.

\section{Effect of Uncertainty on Cost and Reliability}

Fig. 2 illustrates the typical expected cost curve for the least-cost designs of the deterministic formulation and stochastic formulation based on the three different COV values. The most obvious result is that increasing the magnitude of uncertainty results in greater design cost, which is expected given the increased range of influent variability and model parameter uncertainty to be incorporated for producing a reliable treatment plant design. The most expensive influent conditions to treat are generally in the influent concentration range of $20-30 \mathrm{mg} / \mathrm{L}$. The region of rapidly increasing costs (less than $20 \mathrm{mg} / \mathrm{L}$ ) is typically associated with contact and, to a lesser extent, direct filtration configurations. The peak, and decreasing, regions of cost are associated with the conventional treatment region. With respect to the individual process constraints, only the flocculation residence time ever results in an active constraint. Thus, neither the sedimentation retention time nor the filter loading rate constraints are important for determining the least-cost design.

Fig. 3 illustrates the effect of increasing variability on the distribution of effluent concentration values for an influent size range

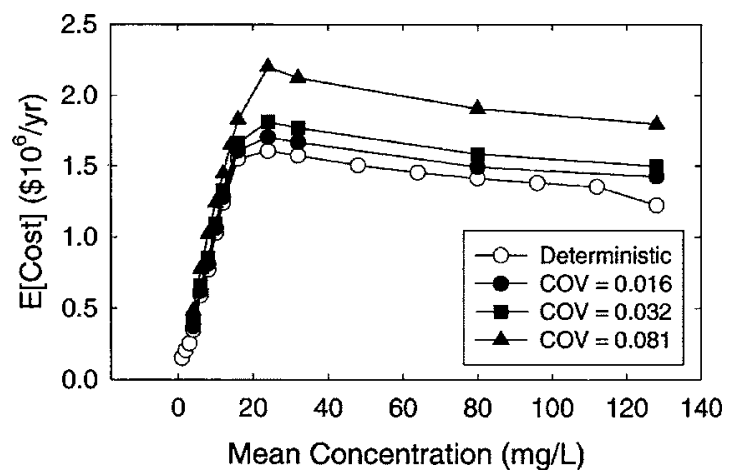

Fig. 2. Expected least-cost values for optimal treatment plant designs over a range of mean influent concentration values for increasing magnitude of variance [influent particle size range of $0.25-25.0 \mu \mathrm{m}$ and $E[\beta]=4$; cost=capital cost annualized at $8 \%$ over 20 years + annual operation and maintenance cost]

of $0.25-25.0 \mu \mathrm{m}, E[C]=32 \mathrm{mg} / \mathrm{L}$, and $E[\beta]=4$. Increasing the $\mathrm{COV}$ of the variable and uncertain parameters results in larger ranges of simulated effluent concentration values. The solid line represents the effluent constraint value of $0.6 \mathrm{mg} / \mathrm{L}$. The deterministic result lies directly on the regulatory line. For the stochastic optimization results, the effluent concentration constraint is satisfied for $95 \%$ of the simulated samples. With increasing COV, the median effluent concentration value decreases to about $0.2 \mathrm{mg} / \mathrm{L}$. Therefore, when designing for reliability, the equivalent deterministic "target" effluent concentration is lower than the regulatory limit.

Given the information provided, one might ask the question: What is the value of the stochastic solution (VSS)? In an unconstrained formulation, the VSS is defined as $E\left[\operatorname{cost}_{\mathrm{det}}\right]-E\left[\operatorname{cost}_{\mathrm{unc}}\right] \geq 0$, where $E\left[\operatorname{cost}_{\mathrm{det}}\right]$ is the expected cost resulting from simulating variability and uncertainty with an optimal deterministic design, and $E\left[\right.$ cost $\left._{\text {unc }}\right]$ is determined by the resulting least-cost design that explicitly incorporates variability and uncertainty in the decision-making process (Morgan and Henrion 1990). This definition indicates that including uncer-

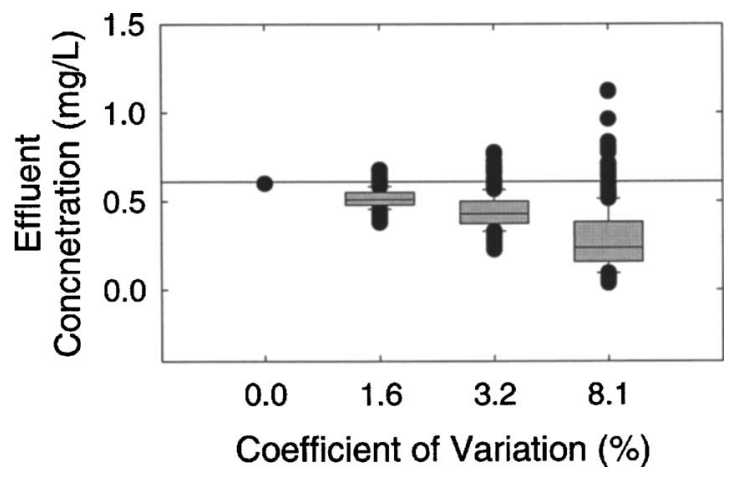

Fig. 3. Box plots for simulated effluent concentration values as a function of increasing COV (size range of $0.25-25.0 \mu \mathrm{m}$, influent concentration of $32 \mathrm{mg} / L, \beta=4$, and $n=300$ ). In the box plots, the middle line represents the median, the box depicts the interquartile range, the lines outside the boxes indicate the $80 \%$ (10-90\%) range, and points beyond these lines are individual simulation results at the tails of the distributions. 


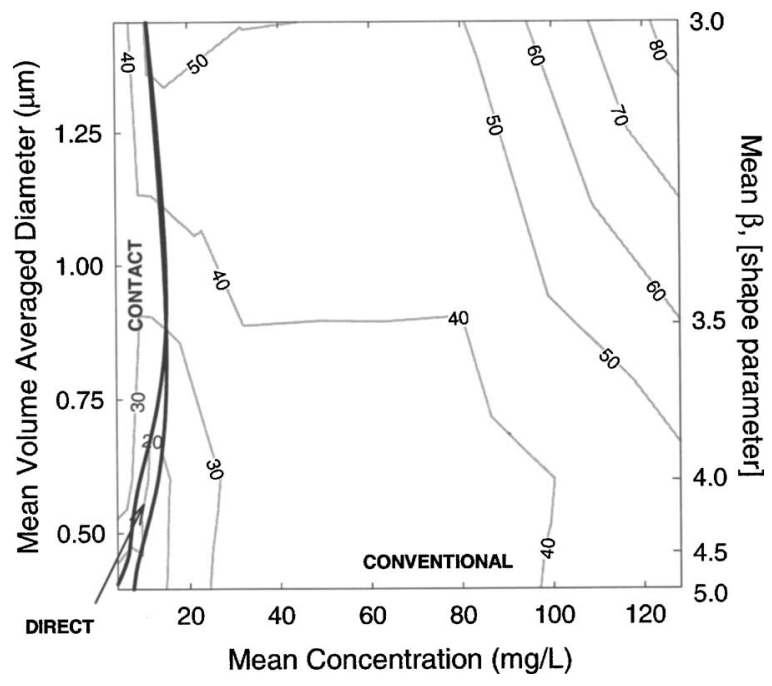

Fig. 4. Least-cost treatment regions and percent increase in expected cost, relative to the deterministic solution, for a range of influent concentrations and $\beta$ values; contours (labeled, gray lines) represent percent cost increase; solid black lines represent the transition between least-cost configurations (influent particle size range $=0.25-25.0 \mu \mathrm{m} ; \beta=3,4$, and $5 ; \mathrm{COV}=0.081)$

tainty in the decision-making process will result in a decision that will cost, at most, no more than when the variable and uncertain information is excluded.

However, the current formulation is a constrained problem, and incorporating uncertainty into the decision-making process results in an increase in cost between 18 and 61\%, as shown in Fig. 2. This increase in cost results from changes in the decisions necessary to provide reliable treatment due to the increased range of effluent particulate concentration (Fig. 3). While the deterministic designs always cost less, simulating the effect of variability and uncertainty results in almost $50 \%$ of the effluent particulate concentration samples violating the effluent concentration constraint. By explicitly incorporating variability and uncertainty in the design process, the design costs increase, however, the effluent concentration constraint violations are reduced to $5 \%$. Therefore, the value of the constrained stochastic solution is not in reducing the cost; the value is in satisfying the effluent concentration constraint with a specified degree of reliability.

\section{Effect of Uncertainty on Least-Cost Treatment Regions}

While some of the specific implications of including variability and uncertainty have been discussed, the overall effect on the least-cost treatment configuration regions is of primary interest. Fig. 4 illustrates: (1) the least-cost regions for contact, direct, and conventional treatment; and (2) the percent increase in cost, relative to the deterministic solution, for $\mathrm{COV}=0.081$ for all variable and uncertain parameters as a function of the mean volumeaveraged particle diameter $\left(\bar{d}_{p}\right)_{V}$ and influent concentration. The solid black lines separate the least-cost regions into contact, direct, and conventional treatment regions. As the preferred treatment option, contact filtration occurs at low influent concentrations, and direct filtration is specified in only a narrow region of low influent concentrations with smaller mean volume-averaged particle diameter. The contour lines (labeled, gray lines) represent the increase in cost due to the incorporation of variability and uncertainty relative to the deterministic solutions. The greatest

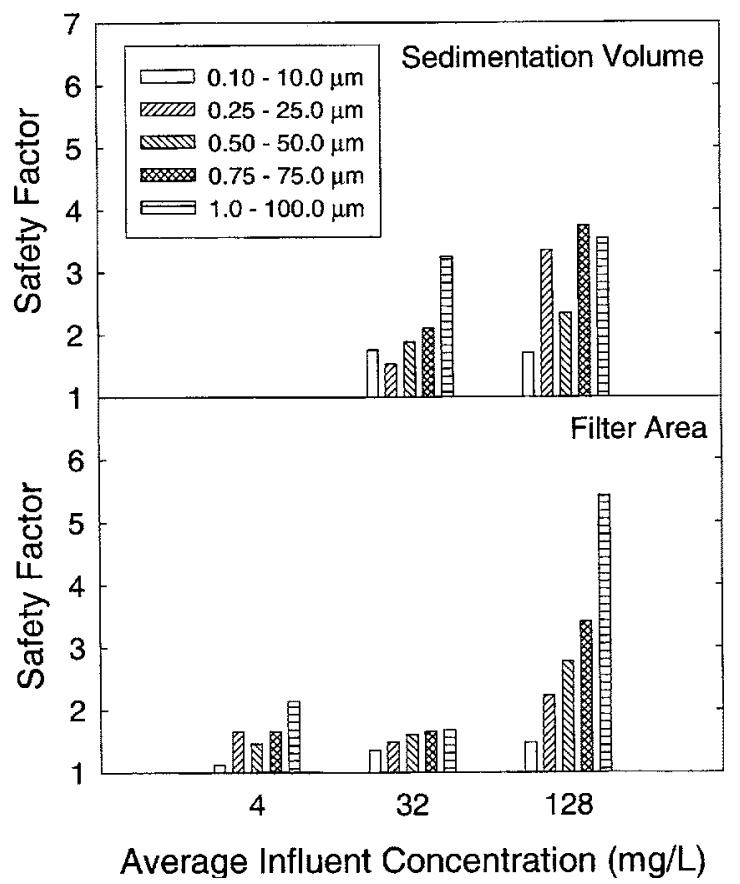

Fig. 5. Equivalent safety factor values for converting the sedimentation volume and filter area of the deterministic solution into the associated optimal stochastic solution for three influent concentrations and each of the five particle size ranges $(\mathrm{COV}=0.081)$

increase in cost occurs for larger $\left(\bar{d}_{p}\right)_{V}$ and at higher influent concentrations. The transition regions between least-cost configurations have the lowest percent increase in cost as a result of: (1) the transition regions being generally the most expensive conditions to treat, even with the deterministic solutions; and (2) one treatment configuration becoming too expensive, causing the least-cost treatment configuration to transition to a different, lower-cost, configuration. In the large particle, high influent concentration regions, just the opposite occurs as this is the least costly region in the deterministic scenarios and there are no other configurations available for treating the water.

These increases in cost are generally associated with increases in sedimentation volume and filter area to provide adequate particulate removal under variable and uncertain conditions. A simplified design approach might be to develop a deterministic design and then apply "safety factors" to adjust the design to provide reliable treatment under variable and uncertain conditions. Ideally, a single safety factor could be applied to an individual process without resulting in an excessively overdesigned process. Fig. 5 presents the equivalent safety factors (i.e., multipliers to convert the deterministic solution to an equivalent design incorporating variability and uncertainty) for sedimentation volume and filter area with mean influent concentrations of 4 , 32 , and $128 \mathrm{mg} / \mathrm{L}$ and $\beta=4$ for the five different particle size ranges (for $C=4 \mathrm{mg} / \mathrm{L}$, sedimentation is not included in the process stream). For filter area with $C=4$ and $32 \mathrm{mg} / \mathrm{L}$, a single safety factor could be applied without excessive overdesign of the process. However, for filter area with $C=128 \mathrm{mg} / \mathrm{L}$ and sedimentation volume, there is much greater variability in the safety factors, suggesting a single safety factor for each process might not be adequate for developing least-cost designs based on deterministic decisions over a range of influent conditions. The current approach of developing safety factors is different than the design 
approach, and safety factors, presented by Kawamura (2000). Using the approach from Kawamura (2000), process characteristics (e.g., loading rate) are selected a priori, which may include a safety factor, and the resulting process capacity is based on maximum flow rates (typically, assumed to be $1.5 \times$ the average flow rate - an additional safety factor) with subsequent process constraint values checked at both average and maximum flow rates to ensure that the process characteristics are appropriate based on experience. This approach implicitly considers the water quality process performance and produces a relatively constant safety factor by simply accommodating changes in hydraulic capacity without explicitly considering design cost. Rather than specify the process characteristics, the current approach constrains the process characteristics [Eqs. (6)-(13)] and explicitly considers changes in water quality via the individual process models when estimating the least-cost design. Given the differences for incorporating water quality (implicit versus explicit, and uncertainty in process models), direct comparisons of the two types of safety factors are not appropriate.

In general, the safety factors exhibit an increasing trend with respect to the influent particle size range. While there are multiple variable and uncertain parameters considered, the increasing safety factors result, in part, from the larger range of simulated particle size distribution as the variability in $\beta$ has greater effects on the larger influent particle size range. For filter area with $C=4$ and $32 \mathrm{mg} / \mathrm{L}$, a single safety factor could be applied without excessive overdesign of the process. However, for filter area with $C=128 \mathrm{mg} / \mathrm{L}$ and sedimentation volume, there is much greater variability in the safety factors, suggesting a single safety factor for each process might not be adequate for developing least-cost designs based on deterministic decisions over a range of influent conditions. While there are many factors included in the design decisions, the relatively constant increase in safety factors for sedimentation with $C=32 \mathrm{mg} / \mathrm{L}$ and filtration with $C=128 \mathrm{mg} / \mathrm{L}$ are a function of the trade-offs between providing additional particulate removal through sedimentation or filtration. There are essentially infinite combinations of sedimentation and filtration designs that provide reliable particulate removal. However, the design decisions associated with sedimentation and filtration are based on a cost and performance trade-off. For $C=32 \mathrm{mg} / \mathrm{L}$, the least-cost design for including variability and uncertainty increases the emphasis on sedimentation. Relative to the deterministic solutions, the sedimentation process is designed, on average, to provide more particulate removal resulting in less particulate matter and larger $\left(\bar{d}_{p}\right)_{V}$ reaching the filter, both of which improve filter performance. For $C=32 \mathrm{mg} / \mathrm{L}$, sedimentation plays an important role in accommodating the variability and uncertainty in the design process. For $C=128 \mathrm{mg} / \mathrm{L}$, the least-cost design places a slightly greater emphasis on filtration. Relative to the deterministic solution, the particulate concentration and $\left(\bar{d}_{p}\right)_{V}$ in the filter effluent are, on average, similar to the deterministic solutions. However, the filter areas for the deterministic solution are relatively small and require significant increases in size to adequately accommodate the impacts of influent variability and uncertainty on the particulate characteristics to be treated, which lead to large safety factors. Such a result further supports the benefits of explicitly including variability and uncertainty in the decision-making process.

Fig. 6 presents the impacts of variability and uncertainty on the least-cost treatment configuration regions for influent size ranges of $0.25-25.0$ and $0.75-75.0 \mu \mathrm{m}$. For the $0.25-25.0 \mu \mathrm{m}$ range [Fig. 6(a)], all three treatment configurations are present.
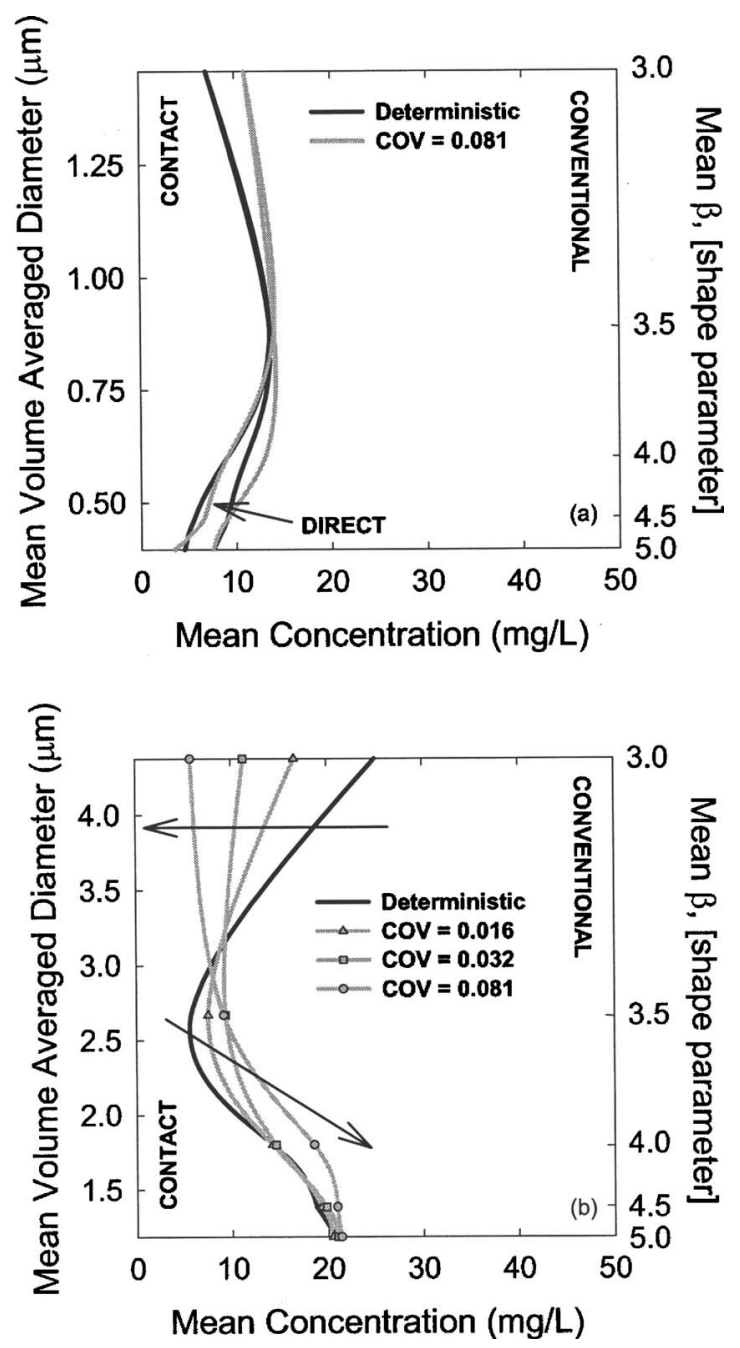

Fig. 6. Least-cost treatment configurations for influent size ranges of: (a) $0.25-25.0 \mu \mathrm{m}$; (b) $0.75-75.0 \mu \mathrm{m}$ as a function of mean volume averaged particle diameter and influent concentration (arrows represent increasing variability; $\mathrm{COV}=0,0.016,0.032$, and 0.081 )

When incorporating the largest COV (0.081) for all variable and uncertain parameters in the design process, there is little shift in the least-cost treatment configuration regions, especially for $\left(\bar{d}_{p}\right)_{V} \leq 1 \mu \mathrm{m}$. For larger influent $\left(\bar{d}_{p}\right)_{V}$, including variability and uncertainty decreases the region where conventional filtration is the least-cost configuration. For the $0.75-75.0 \mu \mathrm{m}$ range [Fig. 6(b)], the effect of the COV assumed for all variable and uncertain parameters on the treatment configuration regions is greater, although only contact and conventional filtration regions are present. For larger $\left(\bar{d}_{p}\right)_{V}$ values, increasing the COV increases the regions where conventional filtration is specified as the leastcost treatment option. For $1.5 \leq\left(\bar{d}_{p}\right)_{V} \leq 2.5 \mu \mathrm{m}$, increasing the $\mathrm{COV}$ decreases the region of conventional filtration.

For Fig. 6(b), a cursory analysis suggests that the shift in leastcost treatment region (conventional $\rightarrow$ contact with increasing variability) is related to the increased size distribution of particle sizes for $\beta=3$ relative to $\beta=4$. For the data points presented, the standard deviation of $\left(\bar{d}_{p}\right)_{V}$ leaving the flocculation process ranges from 0.23 to $1.19 \mu \mathrm{m}$ for $\beta=3$ and from 0.07 to $0.44 \mu \mathrm{m}$ for $\beta=4$ with increasing $\mathrm{COV}$. In part, the increased range of $\left(\bar{d}_{p}\right)_{V}$ increases the filter area necessary to maintain effective 
contact filtration. This increase in filter area can be reduced by the addition of sedimentation through conventional treatment. However, simply considering $\left(\bar{d}_{p}\right)_{V}$ is only part of the reason for the large shift in least-cost regions for $\beta=3$. In addition to the variability in $\left(\bar{d}_{p}\right)_{V}$, the average value of $\left(\bar{d}_{p}\right)_{V}$ is larger for $\beta=3$ (4.44 $\mu \mathrm{m}$ versus $1.87 \mu \mathrm{m}$ for $\beta=4)$. From a removal standpoint, the influent with a smaller $\left(\bar{d}_{p}\right)_{V}$ is more difficult to treat during both sedimentation and filtration [filtration removal efficiency is a minimum near $\left.\left(\bar{d}_{p}\right)_{V}=1.0 \mu \mathrm{m}\right]$. For $\beta=3$, the larger shifts in leastcost treatment regions occur because sedimentation provides significant removal $(52-80 \%)$ without reducing the $\left(\bar{d}_{p}\right)_{V}$ to sizes much more difficult to remove through filtration [20-31\% decrease in $\left.\left(\bar{d}_{p}\right)_{V}\right]$. For $\beta=4$, the conditions are less favorable for a trade-off between sedimentation and filtration. In general, sedimentation still provides significant removal (38-60\%), yet the $\left(\bar{d}_{p}\right)_{V}$ entering the filter remains relatively unchanged [6-9\% increase in $\left.\left(\bar{d}_{p}\right)_{V}\right]$. For $\beta=3$, the removal through sedimentation reduces the loading to the filter to a such a degree that even the lower filter efficiency [based on $\left(\bar{d}_{p}\right)_{V}$ ] allows a cost trade-off between sedimentation and filtration to be realized. For $\beta=4$, the sedimentation process has less impact on the mass loading to the filter and provides little performance improvement by altering $\left(\bar{d}_{p}\right)_{V}$. These results illustrate that incorporating variability and uncertainty into the decision-making process does not result in uniform shifts of the least-cost treatment regions for the different influent particle size ranges considered.

\section{Important Variable and Uncertain Distributions}

The incorporation of variability and uncertainty in the design process provides information that can be used to determine the relative importance of the individual parameter distributions for reliably satisfying the effluent particulate concentration constraint. This is accomplished here using the generalized sensitivity method (Spear and Hornberger 1980). To test the relative importance of an individual variable or uncertain parameter, the simulated sample is first separated into two categories associated with a simulated effluent concentration: (1) "violation," if $C_{\text {eff }}>0.6 \mathrm{mg} / \mathrm{L}$; or (2) "nonviolation," if $C_{\text {eff }} \leq 0.6 \mathrm{mg} / \mathrm{L}$. The resulting distributions of the violation and nonviolation subsets are compared using the Kolmogorov-Smirnov two-sample test (DeGroot and Schervish 2002). If the violation and nonviolation distributions, $G(x)$ and $F(x)$, respectively, are statistically different, the associated variable or uncertain parameter may be considered important for reliably satisfying the effluent concentration constraint.

The Kolmogorov-Smirnov two-sample test statistic is the maximum difference between the two empirical cumulative distribution functions, $D_{m, n}=\sup \left|F_{m}(x)-G_{n}(x)\right|,-\infty<x<\infty$. In this case, the comparison is between the distribution of violated $\left(n_{\text {viol }}=15\right)$ and nonviolated $\left(n_{\text {nonviol }}=285\right)$ samples for satisfying the effluent concentration constraint, which is based on a total sample size of $n=300$ with $5 \%$ of the samples allowed to exceed the effluent concentration. The importance of the individual parameter distributions may be classified in one of three ways, based on Spear and Hornberger (1980):

1. Critical: indicates a $>99 \%$ confidence that the distributions are different; the maximum distance between the two distributions, $D_{m, n}$, must be $\geq 0.433$.

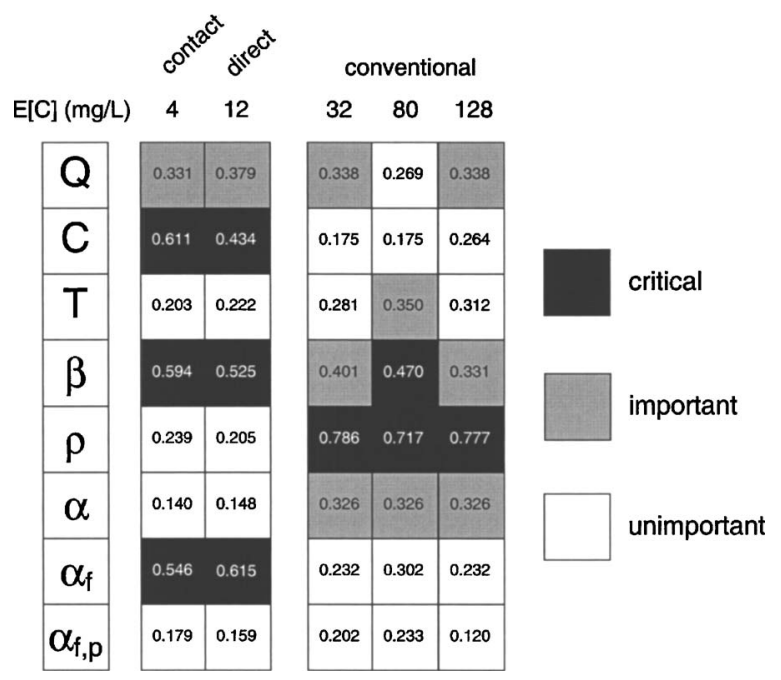

Fig. 7. Individual boxes indicate the Spear and Hornberger (1980) classification: unimportant (white; $D_{m, n} \leq 0.325$ ); important (gray; $0.325 \leq D_{m, n} \leq 0.433$ ); or critical (black; $D_{m, n} \geq 0.433$ ) for the five variable and three uncertain parameters with an influent size range of $0.25-25.0 \mu \mathrm{m}$ and mean $\beta=4\left(D_{m, n}\right.$ values are presented in each box)

2. Important: indicates a 90-99\% confidence that the distributions are different; the maximum distance between the two distributions, $D_{m, n}$, must be between 0.325 and 0.433 .

3. Unimportant: indicates a $<90 \%$ confidence that the distributions are different; the maximum distance between the two distributions, $D_{m, n}$, must be $\leq 0.325$.

Fig. 7 presents the "critical" and "important" parameter distributions, and $D_{m, n}$ values, for satisfying the effluent concentration constraint for five different expected influent concentrations, a $\mathrm{COV}=0.081$ for all variable and uncertain parameters, $\beta=4$, and an influent size range of $0.25-25.0 \mu \mathrm{m}$. The critical parameters strongly follow the primary removal mechanism. For contact and direct filtration, where filtration is the only removal mechanism, the uncertainty in $\alpha_{f}$ is determined to be critical. For conventional filtration, sedimentation typically removes the majority of particulate matter, hence, $\rho$ is critical as particulate density affects the settling rate. Contact and direct filtration are also observed to be sensitive to multiple variable and uncertain distributions. In addition to the uncertainty in $\alpha_{f}$, variability in the influent concentration and shape parameter [which directly affects the influent $\left.\left(\bar{d}_{p}\right)_{V}\right]$ are also critical to the removal of particulate matter.

Other parameters are either less closely related to the objective of reliably satisfying the effluent particulate concentration limit, but still influential (important), or apparently uninfluential in determining whether the objective is met (unimportant). In most cases, flow rate variability is shown to be important; thus, effects on residence time and filter loading rate are important to the success of particulate removal for the given designs. For conventional treatment, in addition to the critical nature of $\rho$, the importance of the shape parameter $\beta$ variability and collision efficiency uncertainty, and the unimportance of filter media removal efficiency, $\alpha_{f, p}$, suggest that the flocculation and sedimentation processes are more sensitive when designing to provide reliable particulate removal.

This analysis provides a decision maker with information related to the importance of the individual parameter variability and 
uncertainty distributions, not the individual parameter (as all parameters in the model affect the effluent concentration). Therefore, these results provide insight into which parameters might be better characterized through additional field or bench-scale studies to improve the representation of system behavior.

\section{Summary and Discussion}

In order to develop robust designs, decision-making with respect to environmental processes must include the inherent variability associated with environmental conditions and the uncertainty in the model parameters used to describe critical processes. This study extends previous research in treatment plant design for particulate removal by developing a decision support tool that explicitly considers variability and uncertainty in the decisionmaking framework. The objective is to determine the appropriate least-cost treatment configuration (contact, direct, or conventional filtration) while satisfying multiple constraints, which include satisfying the effluent particulate concentration constraint at a $95 \%$ confidence level.

In general, increasing the magnitude of the variability and uncertainty increases the expected design cost as the designs are forced to satisfy a successively lower expected effluent particulate concentration to maintain reliable treatment. Ultimately, the value of the stochastic solution is not in the cost, rather the value is in satisfying the regulatory effluent concentration constraint at the targeted confidence level. While the associated sedimentation and filtration design parameters increase to accommodate the variability and uncertainty, the magnitude of these design-variable increases, relative to deterministic least-cost solutions, are dependent on the influent conditions. A wide range of equivalent safety factors (to accommodate variability and uncertainty based on deterministic designs) is determined, and indicates a single safety factor would not always result in least-cost designs, and might not provide reliable treatment.

With respect to overall design, the inclusion of variability and uncertainty has different effects on the least-cost configuration regions. For a larger influent particulate size range, increasing the magnitude of variance and uncertainty expands the conventional treatment region for larger influent particle sizes [influent $\left.\left(\bar{d}_{p}\right)_{v}>3 \mu \mathrm{m}\right]$ and decreases the conventional treatment region for a range of smaller particle sizes $[1.5<$ influent $\left.\left(\bar{d}_{p}\right)_{v}<2.5 \mu \mathrm{m}\right]$. For a smaller influent particulate size range, the inclusion of variability and uncertainty has little effect on the configuration regions.

While all of the parameters have an effect on the effluent concentration, the information generated in the solution framework allows the determination of the relative importance of the individual parameter distributions with respect to reliably satisfying the effluent concentration constraint. In general, critical parameters follow the primary removal mechanism, and contact and direct filtration appear more sensitive than conventional filtration to input variability and uncertainty when reliably satisfying the effluent particulate concentrations constraint. Surprisingly, influent concentration variability and filter media removal efficiency uncertainty are found to be relatively unimportant for conventional treatment. This additional information can be used to determine future resource expenditures to better characterize the parameters through additional laboratory or field studies.

While these computational approaches have had some success
(Wiesner et al. 1987), in general, these process design frameworks are not appropriate for making design decisions in the drinking water treatment area. Rather, these approaches are best suited to investigate factors that may impact design decisions or as screening tools rather than replacements for bench- and pilotscale studies. While the current application is limited by only considering a single contaminant and minimal treatment processes, the framework is sufficiently generalizable to be extended for multiple contaminants and additional process models, including residual handling processes. However, in doing so, one must consider the potential effects of model uncertainty when interpreting the results. For many treatment processes, multiple process models may be available (e.g., rectilinear, curvilinear, and fractal approaches for particle size distribution dynamics) that may incorporate different mechanisms and different model parameters. Comparisons of reliability-based designs obtained with different process models might thus be a useful next step in this research. The framework can also be extended to consider additional constraints that arise when designing an actual water treatment plant, such as limiting the footprint of selected processes based on the available area for constructing the plant.

\section{Acknowledgments}

This research was performed while one of the writers (D.L.B.) was a doctoral student in the Department of Civil and Environmental Engineering at Carnegie Mellon University. Thanks are due to Lorenz Biegler and Jared Cohon for the help that they provided to D.L.B. in his research. The writers wish to acknowledge the NSF Graduate Research Traineeship in Civil Infrastructure Systems, Grant No. GER-9553380, for providing financial support for this research. Partial funding for this work was also provided by the U.S. Environmental Protection Agency, Grant No. CR825188-01-3. It has not been subject to the agency's review and, therefore, does not necessarily reflect the views of the agency, and no official endorsement should be inferred.

\section{References}

Acevedo, J., and Pistikopoulos, E. N. (1998). "Stochastic optimizationbased algorithms for process synthesis under uncertainty." Comput. Chem. Eng., 22(4/5), 647-671.

Amirtharajah, A., and O'Melia, C. R. (1990). "Coagulation processes: Destabilization, mixing, and flocculation." Water quality and treatment, McGraw-Hill, New York, 269-365.

Biegler, L. T., and Cuthrell, J. E. (1985). "Improved infeasible path optimization for sequential modular simulators. II: The optimization algorithm." Comput. Chem. Eng., 9(3), 257-267.

Boccelli, D. L. (2003). "A decision support tool for the design of integrated treatment systems for drinking water utilities." Ph.D. thesis, Carnegie Mellon Univ., Pittsburgh.

Boccelli, D. L., Small, M. J., and Diwekar, U. M. (2004). "Treatment plant design for particulate removal: Effects of flow rate and particle characteristics." J. Am. Water Works Assoc., 96(11), 77-90.

Chaudhuri, P. D., and Diwekar, U. M. (1996). "Process synthesis under uncertainty: A penalty function approach." AIChE J., 42(3), 742-752.

Clark, R. M. (1982). "Cost estimating for conventional water treatment." J. Envir. Engrg. Div., 108(EE5), 819-834.

de Hoop, M. V., and Spencer, C. (1996). "Quasi-Monte Carlo integration over $S^{2} \times S^{2}$ for migration $\times$ inversion." Inverse Probl., 12, 219-239.

DeGroot, M. H., and Schervish, M. J. (2002). Probability and statistics, 3rd Ed., Addison-Wesley, Boston. 
Dharmappa, H. B., Fujiwara, O., Verink, J., and Vigneswaran, S. (1994). "Water-treatment-system design for turbidity removal. II: Optimization." J. Environ. Eng., 120(4), 921-942.

Diwekar, U. M., and Kalagnanam, J. R. (1997). "Efficient sampling technique for optimization under uncertainty." AIChE J., 43(2), 440-447.

Eldred, M. S., Giunta, A. A., Wojtkiewicz, S. F., Jr., and Trucano, T. G. (2002). "Formulations for surrogate-based optimization under uncertainty." Proc., 9th AIAA/ISSMO Symp. on Multidisciplinary Analysis and Optimization, Atlanta, Paper AIAA-2002-5585.

Han, M., and Lawler, D. F. (1991). "Interactions of two settling spheres: Settling rates and collision efficiency." J. Hydraul. Eng., 117(10), 1269-1289.

Han, M., and Lawler, D. F. (1992). "The (relative) insignificance of $G$ in flocculation." J. Am. Water Works Assoc., 84(10), 79-91.

Hindmarsh, A. C. (1983). "A systematized collection of ODE solvers." Scientific computing: Applications of mathematics and computing to the physical sciences, R. S. Stepleman, ed., North-Holland, Amsterdam, The Netherlands, 55-64.

J. M. Montgomery. (1985). Water treatment principles and design, WileyInterscience, New York.

Kawamura, S. (2000). Integrated design and operation of water treatment facilities, 2nd, Ed., Wiley, New York.

Lawler, D. L., O’Melia, C. R., and Tobiason, J. E. (1980). "Integral water treatment plant design: From particle size to plant performance." Particulates in water: Characterization, fate, effects, and removal, M. C. Kavanaugh and J. O. Leckie, eds., American Chemical Society, Washington, D.C., 353-388.

Lee, D. G., Bonner, J. S., Garton, L. S., Ernest, A. N. S., and Autenrieth, R. L. (2000). "Modeling coagulation kinetics incorporating fractal theories: A fractal rectilinear approach." Water Res., 34(7), 1987 2000.

Letterman, R. D. (1980). "Economic analysis of granular-bed filtration." J. Envir. Engrg. Div., 106(EE2), 279-291.

Li, X.-Y., and Logan, B. E. (1997). "Collision frequencies of fractal aggregates with small particles by differential sedimentation." Environ.
Sci. Technol., 31(4), 1229-1236.

Li, X.-Y., and Logan, B. E. (2000). "Settling and coagulating behavior of fractal aggregates.” Water Sci. Technol., 42(3), 253-258.

Mhaisalkar, V. A., Bassin, J. K., Paramasivan, R., and Khanna, P. (1993). "Dynamic programming optimization of water-treatment-plant design." J. Environ. Eng., 119(6), 1158-1175.

Morgan, M. G., and Henrion, M. (1990). Uncertainty, Cambridge University Press, Cambridge, U.K.

O'Melia, C. R., and Ali, W. (1978). "The role of retained particles in deep bed filtration." Prog. Water Technol., 10(5/6), 167-182.

Pistikopoulos, E. N. (1995). "Uncertainty in process design and operations." Comput. Chem. Eng., 19(Supp. 1), 553-563.

Ramaley, B. L., Lawler, D. F., Wright, W. C., and O'Melia, C. R. (1981). "Integral analysis of water plant performance." J. Envir. Engrg. Div., 107(EE3), 547-562.

Spear, R. C., and Hornberger, G. M. (1980). "Eutrophication in Peel Inlet. II. Identification of critical uncertainties via generalized sensitivity analysis." Water Res., 14(1), 43-49.

Straub, D. A., and Grossman, I. E. (1993). "Design optimization of stochastic flexibility." Comput. Chem. Eng., 17(4), 339-354.

U.S. Environmental Protection Agency (USEPA). (1998). "Interim enhanced surface water treatment rule." Federal Register 69478-69521, Washington, D.C.

Wiesner, M. R. (1985). "Optimal water treatment plant configuration: Effects of raw water characteristics." Ph.D. thesis, Johns Hopkins Univ., Baltimore.

Wiesner, M. R. (1992). "Kinetics of aggregate formation in rapid mix." Water Res., 26(3), 379-387.

Wiesner, M. R., and Mazounie, P. (1989). "Raw water characteristics and the selection of treatment configurations for particulate removal." J. Am. Water Works Assoc., 81(5), 80-89.

Wiesner, M. R., O’Melia, C. R., and Cohon, J. L. (1987). "Optimal water treatment plant design." J. Environ. Eng., 113(3), 567-584.

Wu, M.-Y., and Chu, W.-S. (1991). "System analysis of water treatment plant in Taiwan." J. Water Resour. Plann. Manage., 117(5), 536-548. 
Copyright of Journal of Environmental Engineering is the property of American Society of Civil Engineers and its content may not be copied or emailed to multiple sites or posted to a listserv without the copyright holder's express written permission. However, users may print, download, or email articles for individual use. 